Copyright (C1996, American Institute of Aeronautics and Astronautics, Inc.

AIAA Meeting Papers on Disc, July 1996

A9637040, AIAA Paper 96-2820

Dynamic response of an industrial turbomachinery rotor

\author{
Marlin Kruse \\ Michigan Univ., Ann Arbor \\ Christophe Pierre \\ Michigan Univ., Ann Arbor
}

\begin{abstract}
AIAA, ASME, SAE, and ASEE, Joint Propulsion Conference and Exhibit, 32nd, Lake Buena Vista, FL, July 1-3, 1996
\end{abstract}

\begin{abstract}
This paper investigates the effects of random blade mistuning on the dynamics of an advanced gas turbine rotor. Both free and forced responses of the rotor are examined using the FEM, and a computationally inexpensive reduced-order modeling technique based on component mode synthesis. The spatially extended free modes of vibration of the tuned rotor are found to undergo severe localization upon the introduction of blade mistuning. In turn, this results in dramatic displacement and stress amplitude increases in the forced response of individual blades. The mistuned forced response amplitude is found to vary considerably with mistuning strength and the degree of aerodynamic and disk structural coupling between the blades. The paper concludes with a statistical study in which Weibull distributions are used to calculate approximate forced response statistics. (Author)
\end{abstract}




\title{
DYNAMIC RESPONSE OF AN INDUSTRIAL TURBOMACHINERY ROTOR
}

\author{
Marlin Kruse* and Christophe Pierre ${ }^{\dagger}$ \\ The University of Michigan, Ann Arbor, Michigan
}

\begin{abstract}
This paper investigates the effects of random blade mistuning on the dynamics of an advanced gas turbine rotor. Both free and forced responses of the rotor are examined using the finite element method, and a computationally inexpensive reduced-order modeling technique based on component mode synthesis. The spatially extended free modes of vibration of the tuned rotor are found to undergo severe localization upon the introduction of blade mistuning. In turn, this results in dramatic displacement and stress amplitude increases in the forced response of individual blades. The mistuned forced response amplitude is found to vary considerably with mistuning strength and the degree of aerodynamic and disk structural coupling between the blades. The paper concludes with a statistical study in which Weibull distributions are used to
\end{abstract} calculate approximate forced response statistics.

\section{Introduction}

Dynamic analyses of turbomachinery traditionally assume spatial repetitivity among the individual sectors that comprise the rotor. The cyclic characteristic enables analysts to reduce the computa tional time considerably by modeling a single sector, as shown in Figure 1, rather than modeling the entire blade assembly. However, cyclic symmetry implies that all sectors are identical, that is, that the system is tuned. Prior experience with turbomachinery rotors-see the works of Ewins ${ }^{1,2}$ and Irretie $^{3}$-indicates that tuned analyses are not always accurate.

Small differences in the structural properties of individual blades, due to manufacturing and material tolerances or in-service degradation, often destroy ideal symmetry. These variations are referred to as blade mistuning. The collapse of symmetry may lead to qualitatively different dynamic behavior than that experienced by a perfectly tuned rotor. In particular, mistuning may inhibit vibration transmission among blades and confine energy to a few, or even a single blade. Mode shapes may become spatially localized and, as a result, a single blade may experience deffections much larger than that

Copyright (C) 1996 by Marlin Jay Kruse. Published by the American Institute of Aeronautics and Astronautics, Inc. with permission.

* Graduate Student Research Assistant, Student Member AIAA.

$\dagger$ Associate Professor, Mechanical Engineering and Applied Mechanics, Senior Member AIAA. predicted by a tuned analysis. 4,5

Mistuning effects must be included in the analysis if accurate predictions of vibratory response amplitudes are to be made. However, analyzing a finite element model of a full blade assembly, such as that shown in Figure 2, is an enormously costly, if not impossible, computational task. The purpose of this paper is to demonstrate, through the case study of an industrial rotor, that mistuned response amplitudes can be accurately and efficiently predicted, in a statistical fashion, by using a novel reduced-order modeling technique. This technique, recently developed by Ottarsson et al. ${ }^{6}$ and Kruse and Pierre, ${ }^{7}$ produces reduced-order models (ROM) of turbomachinery rotors directly from their finite element models. The procedure involves a component mode analysis of the rotor, with a truncated number of modal amplitudes describing the response of the assembly. The key idea introduced by Ottarsson et $a l^{6}$ is that the motion of an individual blade consists of both cantilever blade elastic motion and diskinduced static motion. The principal advantage of the reduced-order modeling technique is the considerable computational savings associated with solving for the dynamic response of an entire mistuned rotor with a reduced set of degrees of freedom.

Another focus of this paper is the use of the ROM to examine the effects of interblade coupling on mistuned forced response amplitudes. It is well known that vibrational energy is transmitted or confined to blades within an assembly according to the magnitude of interblade coupling. ${ }^{5,8}$ In non-shrouded industrial rotors, the two predominant forms of interblade coupling are aerodynamic and disk structural coupling. Aerodynamic coupling results from the interaction of blades with either a gaseous or 


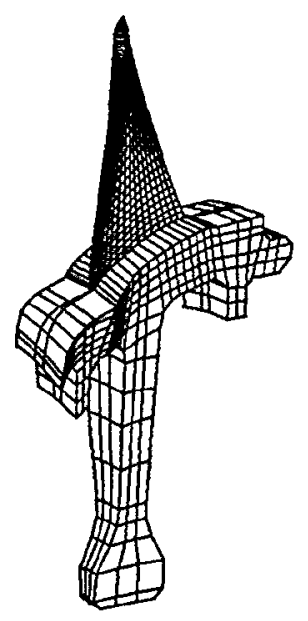

Figure 1: Single-sector structural model of a 29blade compressor rotor stage.

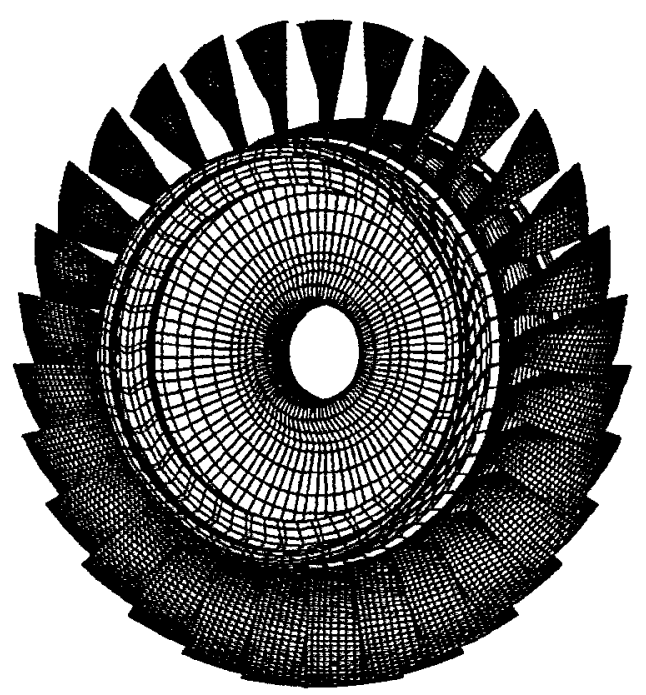

Figure 2: Full structural model of a 29-blade compressor rotor stage. liquid medium, while disk structural coupling represents the transmission of vibrational energy between blades through the disk. One objective of this paper is to examine separately the effects of aerodynamic and disk structural coupling on mistuned dynamic response.

This paper examines the free and forced dynamic responses of an industrial turbomachinery rotor with blade mistuning. In Section 2, the reduced-order modeling formulation is briefly reviewed. Section 3 describes the rotor studied and discusses the $\mathrm{fi}$ nite element and reduced-order models used in the analysis. The free response of the tuned and mistuned rotors is discussed in Section 4, and the effects of aerodynamic and disk structural coupling on the forced response of the mistuned rotor to engine order excitations are examined in Section 5. The paper concludes with a study of the forced response statistics of randomly mistuned rotors.

There are three significant contributions of this work. First, the computationally inexpensive reducedorder model technique introduced by Ottarsson et al. ${ }^{6}$ and Kruse and Pierre ${ }^{7}$ is shown to correlate extremely well with the much larger finite element model of the industrial rotor. Second, levels of aerodynamic and disk structural coupling commonly encountered in practice are shown to produce forced response amplitudes of mistuned rotors that are significantly larger than those of tuned rotors. Third, efficient statistical simulations are carried out, yielding true estimates of the increase in forced response amplitude due to mistuning. Although all three contributions are significant, it is the computational validation of the ROM technique that is of particular importance to the industrial manufacturers of turbomachinery rotors. Prior to the development of this technique, statistical or even single mistuning pattern analyses were impossible to perform for most finite element models of industrial rotors.

\section{A Brief Review of the}

\section{Reduced-Order Model Formulation}

The ROM formulation for bladed disks is based on the idea that the motion of an individual blade consists of cantilever blade elastic motion and diskinduced static motion. ${ }^{6}$ Figure 3 illustrates these two types of blade motion. The finite element displacements of the blade degrees of freedom, $\mathbf{u}$, are thus-expanded as:

$$
\mathbf{u}=\mathbf{U}^{\mathbf{d}} \mathbf{a}+\mathbf{U}^{\mathbf{b}} \mathbf{b}
$$

where $U^{d}$ is a matrix whose columns are the blade portions of the disk-induced mode shapes, $U^{b}$ is a 
matrix whose columns are the mode shapes of the cantilevered blades, and the vectors $a$ and $b$ contain the disk and blade modal coordinates, respectively.

In order to derive the free response ROM from the standard finite element representation of a sector, two finite element models of bladed-disk components are required. The first model is that of a single disk-blade sector. Cyclic symmetry is utilized to determine the disk-induced static mode shapes, $\tilde{\mathbf{u}}_{\mathbf{n}, \mathbf{m}}^{\mathbf{d}}$. Since static blade deformations are sought, the blade elements are massless. The second model is that of a cantilevered blade, leading to the generation of the cantilever blade free vibration mode shapes, $\overline{\mathbf{u}}_{i}^{\mathrm{b}}$.

External excitation can be included in the ROM formulation. Kruse and Pierre ${ }^{7}$ considered a bladed disk subjected to an arbitrary external force vector, F, which may be applied to all the blade degrees of freedom of the assembly. Similarly, motiondependent aerodynamic forces, viscous and structural damping, and blade mistuning can all be incorporated in the ROM formulation. The ROM equations of motion are given by:

$$
\begin{aligned}
& {\left[\begin{array}{cc}
I+B \operatorname{diag}\left[\overline{\mathbf{U}}_{\mathbf{n}}^{\mathbf{d}^{\mathrm{T}}} \overline{\mathbf{M}}_{\mathbf{b}} \overline{\mathbf{U}}_{\mathbf{n}}^{\mathbf{d}}\right] & \mathbf{U}^{\mathbf{d}^{\mathrm{T}} \mathbf{M}_{\mathrm{b}} \mathbf{U}^{\mathrm{b}}} \\
\mathbf{U}^{\mathbf{b}^{\mathrm{T}}} \mathbf{M}_{\mathbf{b}} \mathbf{U}^{\mathbf{d}} & \mathbf{I}
\end{array}\right]\left[\begin{array}{c}
\ddot{a} \\
\ddot{b}
\end{array}\right]} \\
& +\left[\begin{array}{cc}
0 & 0 \\
0 & \operatorname{diag}(2 \zeta) \otimes \sqrt{\hat{\mathbf{K}}_{\mathrm{b}}}
\end{array}\right]\left[\begin{array}{l}
\dot{\mathbf{a}} \\
\dot{\mathbf{b}}
\end{array}\right]+\left(1+j G_{\text {Struc }}\right) \\
& {\left[\begin{array}{cc}
\hat{\mathbf{K}}_{\mathbf{d}}+\mathbf{B d i a g}\left[\tilde{\mathbf{U}}_{\mathbf{n}}^{\mathbf{d}^{\mathrm{T}}} \tilde{\mathbf{K}}_{\mathbf{b}} \tilde{\mathbf{U}}_{\mathbf{n}}^{\mathbf{d}}\right] & \mathbf{U}^{\mathbf{d}^{\mathrm{T}} \mathbf{K}_{\mathbf{b}} \mathbf{U}^{\mathrm{b}}} \\
\mathbf{U}^{\mathbf{d}^{\mathrm{T}}} \mathbf{K}_{\mathbf{b}} \mathbf{U}^{\mathbf{b}} & \operatorname{diag}\left(1+\delta_{i}\right) \otimes \hat{\mathbf{K}}_{\mathbf{b}}+\mathbf{A}_{\mathbf{R O M}}
\end{array}\right]} \\
& {\left[\begin{array}{l}
\mathbf{a} \\
\mathbf{b}
\end{array}\right]=\left[\begin{array}{l}
\mathbf{U}^{\mathbf{d}^{\mathrm{T}} \mathbf{F}} \\
\mathbf{U}^{\mathbf{b}^{\mathrm{T}} \mathbf{F}}
\end{array}\right]}
\end{aligned}
$$

where all quantities are defined in the nomenclature, Appendix A.

\section{Rotor Description} and Computational Models

The industrial rotor, illustrated in Figure 2, is the second stage of a four-drum compressor rotor used in an advanced gas turbine application. The design is referred to as a blisk, since the blades and disk are machined from a single, continuous piece of material. Table 1 lists material properties for the rotor. There are 29 blades in the rotor.

Cyclic symmetry routines in MSC/NASTRAN ${ }^{T M}$ are used to calculate the free vibration natural frequencies and mode shapes, and the forced response to an engine order excitation of the tuned finite element model, by using the single sector representation of Figure 1. The sector model consists of eightnoded solid elements, with a rib located at the outer

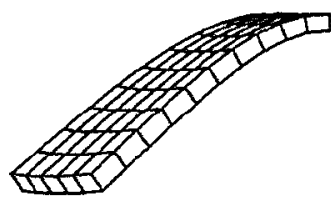

(a)

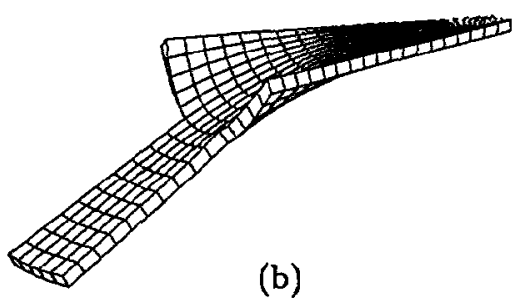

Figure 3: Cantilever blade (a) and disk-induced (b) motions.

edge of the disk constrained to have zero displacement. (In reality, the rib extends on to the next stage of the compressor, so there is slight flexibility at the interface.) The disk portion of the model contains 528 elements, while the blade is modeled with 375 elements. There are 4,374 degrees of freedom per sector in the finite element model. In contrast, the ROM consists of five disk-induced modes and ten cantilever blade modes, for a total of 15 degrees of freedom per sector.

The mistuned finite element model consists of the entire blade assembly, as shown in Figure 2. The same mesh pattern is used in the single-sector cyclic symmetry model and the full mistuned model. Mistuning is introduced into the assembly by allowing each blade to have a different Young's modulus. For the $i$ th blade, one has

$$
E_{i}=E_{0}\left(1+\delta_{i}\right)
$$

where $E_{o}$ is Young's modulus for a tuned blade, and $\delta_{i}$ is the dimensionless mistuning parameter associated with each individual blade. Table 2 lists the mistuning distribution for the industrial rotor. The parameters are based on experimental natural frequency measurements on a prototype rotor. There are 126,846 degrees of freedom in the mistuned finite element model, as compared to 435 degrees of freedom in the mistuned ROM.

\section{Free Response}

It is convenient to describe the mode shapes of a tuned rotor in terms of nodal diameters and nodal circles. Nodal diameters are nodal lines across the diameter of the disk, while nodal circles are nodal 


\begin{tabular}{||c|c||}
\hline \hline Material Property & Property Value \\
\hline Modulus of Elasticity, $E_{o}$ & $29.5(10)^{6} \mathrm{psi}$ \\
Modulus of Rigidity, $G$ & $11.3(10)^{6} \mathrm{psi}$ \\
Mass Density, $\rho$ & $7.40(10)^{-4} \frac{\mathrm{lbsec}^{2}}{\mathrm{in}^{4}}$ \\
Poisson's Ratio, $\nu$ & 0.305 \\
Structural Damping, $G_{\text {Struc }}$ & 0.006 \\
\hline
\end{tabular}

Table 1: Blisk material properties.

\begin{tabular}{||c|c||}
\hline Blade Number & Mistuning $\delta_{i}$ \\
\hline 1 & 0.05704 \\
2 & 0.01206 \\
3 & 0.04670 \\
4 & -0.01502 \\
5 & 0.05969 \\
6 & -0.03323 \\
7 & -0.00078 \\
8 & -0.01688 \\
9 & 0.00242 \\
10 & -0.02746 \\
11 & -0.03630 \\
12 & -0.03569 \\
13 & -0.03630 \\
14 & -0.03630 \\
15 & 0.00242 \\
16 & 0.04934 \\
17 & 0.04478 \\
18 & 0.03030 \\
19 & 0.00242 \\
20 & 0.01733 \\
21 & 0.02919 \\
22 & -0.00328 \\
23 & 0.00085 \\
24 & -0.03653 \\
25 & -0.03630 \\
26 & -0.01665 \\
27 & 0.00782 \\
28 & -0.01168 \\
29 & -0.01331 \\
\hline \hline
\end{tabular}

Table 2: Mistuning pattern for the case study rotor, based on natural frequency measurements on a prototype rotor.

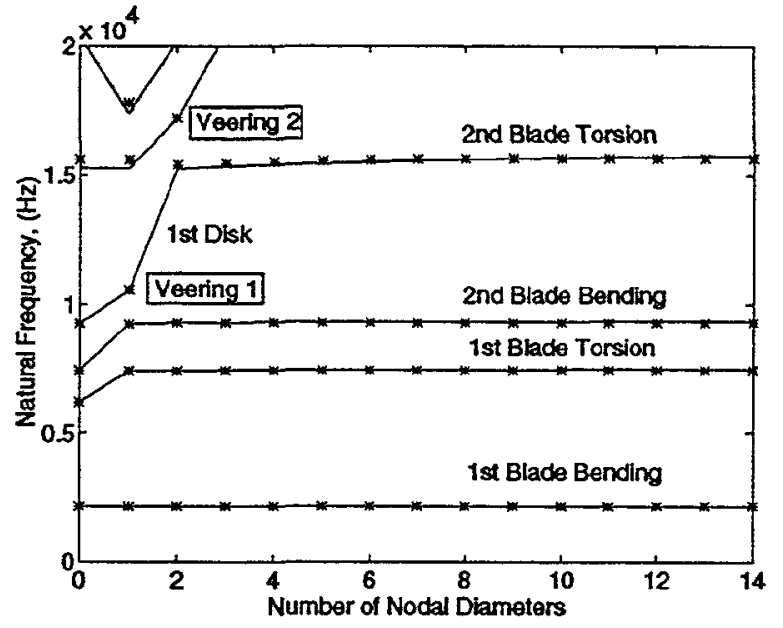

Figure 4: Natural frequencies versus number of nodal diameters, for the tuned rotor in Fig. 2, by finite element (-) and reduced-order (*) modeling. Note the excellent agreement between finite element and ROM natural frequencies.

lines in the circumferential direction. The nodal diameter and nodal circle characteristics of the free vibration modes are conveniently summarized by a plot of natural frequencies versus the number of nodal diameters, as shown in Figure 4. Figure 4 reveals two interesting features of the tuned rotor's modal structure. First, as the number of nodal diameters increases, the disk stiffens rapidly, and the slanted lines in Figure 4 correspond to disk-dominated modes. In the absence of blade tip or mid-span shrouding, the blade-dominated modes do not stiffen significantly as the number of nodal diameters increases. Lines that are approximately horizontal, therefore, represent families of blade-dominated modes. The family of modes around $2,150 \mathrm{~Hz}$, for instance, features motion that is dominated by the first bending mode of a cantilevered blade, while motion in the second family of modes, which is at about 7,400 $\mathrm{Hz}$, is dominated by the first torsional mode of a cantilevered blade. Both of these families of modes exhibit no nodal circles. Motion in the third $(9,100$ $\mathrm{Hz})$ and fourth $(15,500 \mathrm{~Hz})$ families of modes is dominated by the second bending and second torsional modes of a cantilevered blade, respectively, and feature one nodal circle. Figure 4 lists the physical description of the modal families.

A second notable feature in Figure 4 are the two areas, called eigenvalue veerings, in which blade- and disk-dominated families of modes veer away from each other. Physically, eigenvalue veerings indicate 
the degree of coupling between families of disk and blade modes. The strength of a veering is measured by the distance between the natural frequencies and the local curvature in the veering region. ${ }^{9}$ The impact of eigenvalue veerings on the forced response of mistuned rotors will be highlighted in Section 4.

The ROM natural frequencies shown in Fig. 4 are only 1 to $2 \%$ higher than the finite element natural frequencies, demonstrating the accuracy of the reduced-order modeling formulation. Figures 5 and 6 depict example four nodal diameter and one diameter mode shapes, respectively, obtained via finite element and reduced-order models. Motion in the four nodal diameter mode is characterized by the first bending mode of a cantilevered blade, while motion in the one nodal diameter mode is dominated by the second bending mode of a cantilevered blade. Unlike the four nodal diameter mode, the one nodal diameter mode is located in the center of an eigenvalue veering (Veering 1 in Fig. 4). Note that for these mode shapes motion extends throughout the blade assembly. Both of these modes will be referred to in Section 4, in which the forced response of the rotor is discussed.

The above nodal diameter description inherently implies that the mode shapes of the rotor are themselves cyclic, that is, spatially extended. This is true for tuned rotors. However, small blade mistuning may alter mode shapes and cause the concentration of vibrational energy to a single blade-the socalled phenomenon of localization. The observation that the first-order mode shape perturbation due to mistuning is inversely proportional to the difference in the tuned system's natural frequencies, leads to the well known property that the localization of the mode shapes is most accute in frequency regions of high modal density. ${ }^{4}$ From Fig. 4, it appears that all families of blade-dominated modes exhibit high modal density and are therefore susceptible to mode localization upon the introduction of mistuning. Examination of selected mode shapes from the mistuned rotor models, as depicted in Figs. 7-10, indicates extremely localized mode shapes. The ROM mode shapes display the same severe localization, and they correlate very well with the finite element mode shapes.

When mode localization occurs, vibrational energy is largely concentrated in a single blade instead of being spatially distributed throughout the structure; hence, there is a strong likelihood that the forced response of the mistuned structure will greatly exceed the tuned response. Wei and Pierre ${ }^{5}$ and Ottarsson and Pierre ${ }^{8}$ determined, for a singledegree of freedom per sector model of a bladed disk,

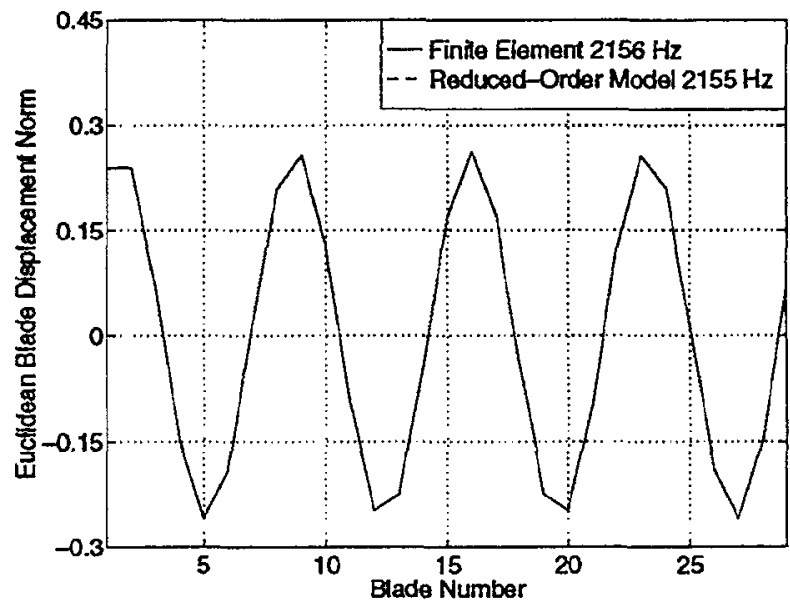

Figure 5: Comparison of tuned finite element and ROM four nodal diameter mode shapes. Motion is characterized by the first bending mode of a cantilever blade.

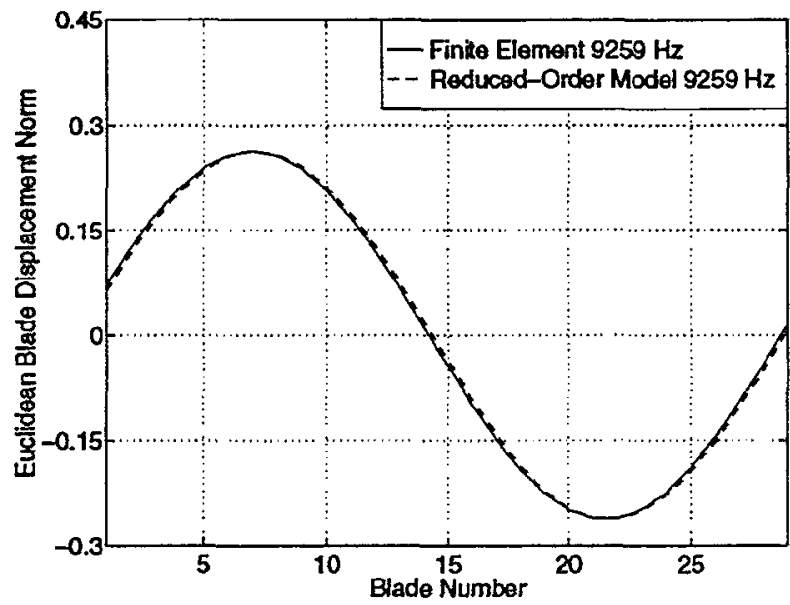

Figure 6: Comparison of tuned finite element and ROM one nodal diameter mode shapes. The natural frequency associated with this mode shape is located in Veering 1 ; and motion is dominated by the second bending mode of a cantilever blade. 


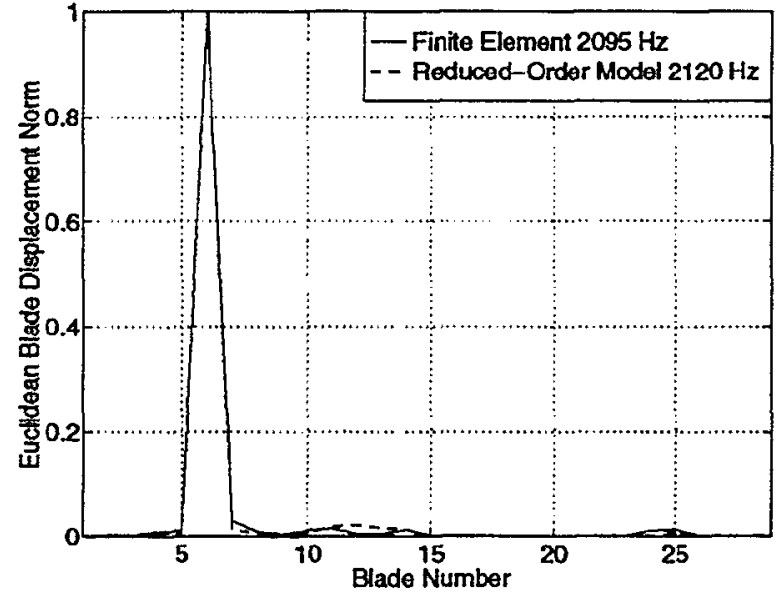

Figure 7: Comparison of mistuned finite element and ROM mode shapes in the frequency region encompassing the first family of blade-dominated modes. The mode shape is spatially localized about blade number six.

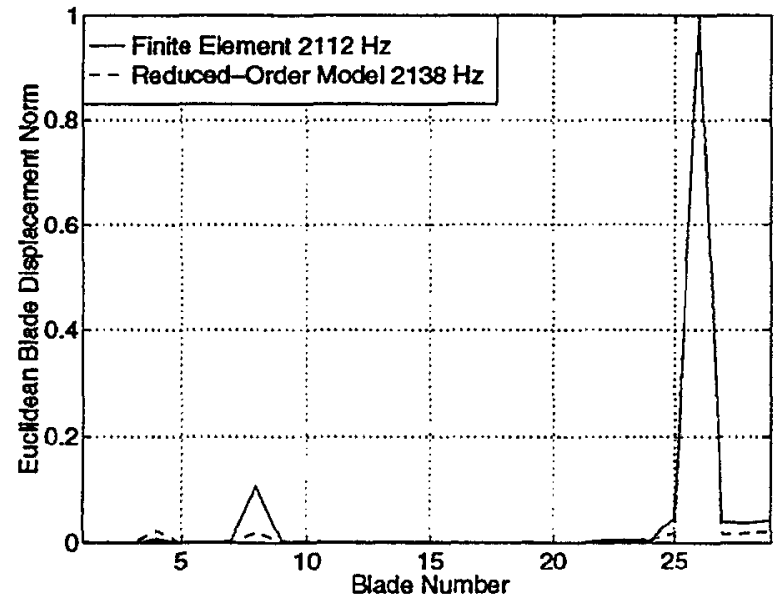

Figure 8: Comparison of mistuned finite element and ROM mode shapes in the frequency region encompassing the first family of blade dominated modes. The mode shape is spatially localized about blade number 26 .

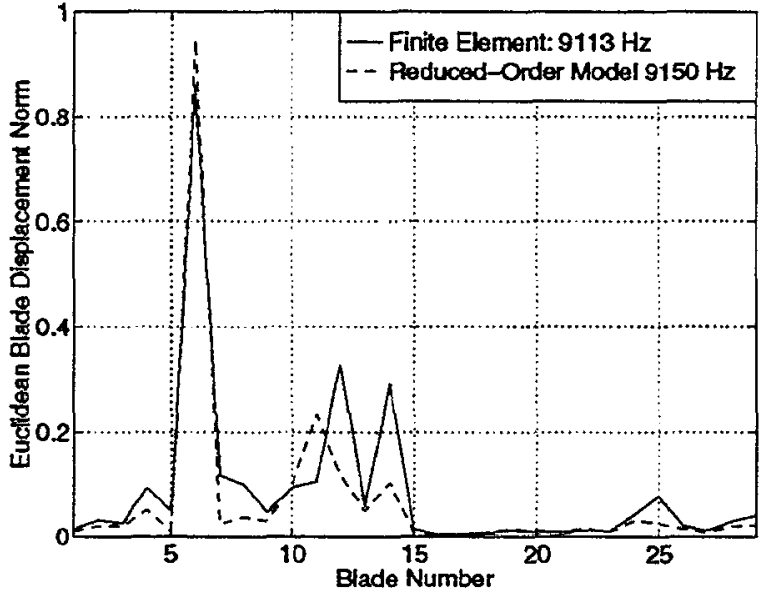

Figure 9: Comparison of mistuned finite element and ROM mode shapes in the first eigenvalue veering. Motion is characterized by the second bending mode of a cantilevered blade and is localized about blade number six.

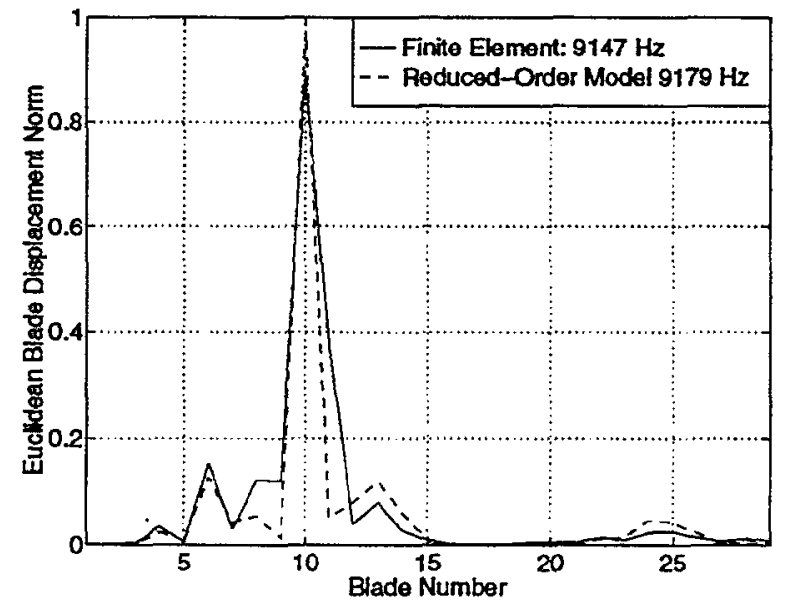

Figure 10: Comparison of mistuned finite element and ROM mode shapes in the first eigenvalue veering- Motion is-characterized by the second bending mode of a cantilevered blade and is localized about blade number ten. 
that moderately weak coupling between blades is required in order for significant amplitude increases in the forced response to occur. If there is no (or very little) interblade coupling, then each blade behaves as an individual mistuned oscillator, and the mistuned response does not deviate significantly from the tuned response. As coupling increases from infinitesimal to moderately weak, an avenue is created for the blades to communicate vibrational energy, which raises the possibility of confining energy to a few blades. The mistuned response then deviates significantly from the tuned response, until further increases in coupling prohibit the confinement of energy, yielding tuned-like response for large coupling values. In the next section, two sources of interblade coupling, aerodynamic and disk structural coupling, are considered separately, and their effects on mistuned blade response to engine order excitation are determined.

\section{Forced Response}

External loading on the case study rotor is assumed to consist of a single unit load located at the tip and mid-chord of each blade, and normal to the surface of the blade. This is a simplified case of the actual loading, where the blades are subject to distributed pressure loads. Pressure loads could be enforced in the ROM by specifying the equivalent nodal loading on all blade degrees of freedom. The excitation is assumed to be harmonic in time and to differ only in phase from blade to blade-the socalled engine order excitation. This type of excitation results from the rotation of the rotor under a stationary force in the flow field, and has been widely used in the literature. ${ }^{10,11}$ The external force vector in Eq. (2) can be expressed in the finite element coordinates as:

$$
\mathbf{F}=e^{j \omega t}\left\{\begin{array}{c}
\tilde{\mathbf{f}} \\
\tilde{\mathbf{f}} e^{j \phi_{1}} \\
\vdots \\
\tilde{\mathbf{f}} e^{j \phi_{N-1}}
\end{array}\right\}
$$

where

$$
\phi_{i}=\frac{2 \pi C(i-1)}{N} \quad i=1, \ldots, 29
$$

is the interblade phase angle of the excitation, $C$ is the engine order excitation, $\tilde{f}$ is the force vector on a single blade, expressed in terms of sector finite element coordinates, and $\mathbf{F}$ is the force vector on the entire assembly of blades. Structural damping, $G_{\text {struc }}$, which was experimentally determined to be 0.006 , is used in both the finite element and ROM forced response calculations. Viscous damping associated with the interaction of blades with a gaseous or liquid medium is introduced in Section 5.1.

\subsection{Aerodynamic Coupling}

Figure 11 depicts the frequency response of the tuned assembly using both finite element and reducedorder models in the frequency region encompassing the first family of blade-dominated modes. Engine order excitation, $C$, is equal to four. A scalar representation of blade deflection amplitude, the displacement norm, is utilized to represent the forced response of the assembly in terms of frequency. The displacement norm is defined as the square root of the sum of the squares of the blade displacement amplitudes for all blade degrees of freedom. In the tuned system response, all blades have identical displacement norms. This is not true, however, for a mistuned rotor, where the various blades feature different response amplitudes. In the mistuned case, the largest blade displacement norm throughout the assembly is selected at each frequency, defining the largest frequency response. Figure 11 shows that the resonant response amplitude of the tuned system using the ROM formulation is only $2 \%$ higher than the corresponding finite element result. Figure 12 depicts a comparison of mistuned finite element and ROM largest frequency responses. Only six mistuned finite element data points are available, due to the tremendous computational expense associated with solving a full blade assembly. Each finite element solution requires approximately $24 \mathrm{cpu}$ hours, 5 GB of disk space, and $270 \mathrm{MB}$ of RAM on a $100 \mathrm{Mhz}$ UNIX workstation. Each ROM solution requires only $33 \mathrm{cpu}$ seconds, $32 \mathrm{MB}$ of disk space, and $13 \mathrm{MB}$ of RAM on a $64 \mathrm{Mhz}$ UNIX workstation. Note that in Figs. 11 and 12, the maximum mistuned response amplitude is only $4 \%$ higher than the tuned resonant amplitude. Recall, from the works of Wei and Pierre ${ }^{5}$ and Ottarsson and Pierre, ${ }^{8}$ that significant amplitude increases occur when there is moderately weak interblade coupling. In this particular frequency region, the mode shapes are dominated by cantilever blade motion, and disk motion is minimal. Individual blades, therefore, are isolated from each other by the disk and cannot effectively communicate energy to one another. The mistuned system thus responds very much like a collection of uncoupled blades, yielding a minimal response amplitude increase due to mistuning. If we introduce aerodynamic coupling between blades, however, an avenue of communication between blades is created, and Fig. 13 shows that the mistuned response may exceed the tuned response by as much as $61 \%$. 


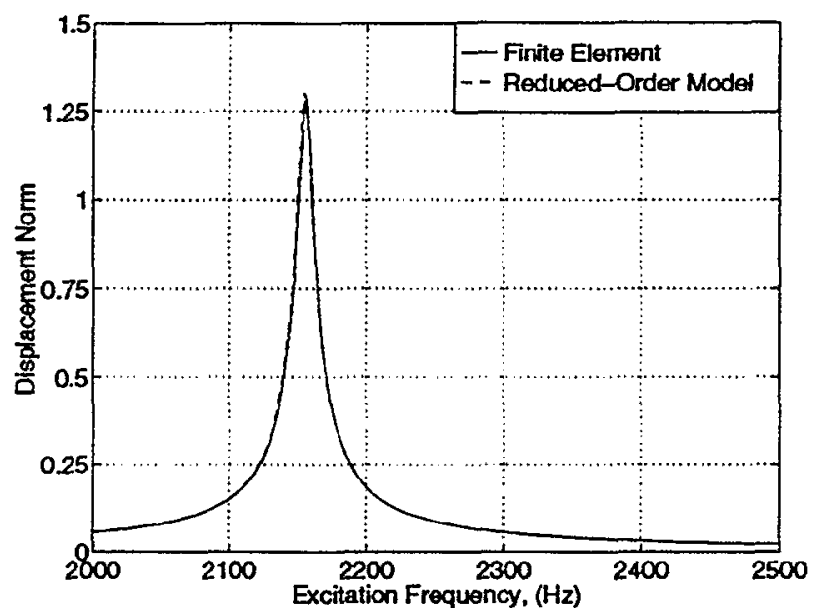

Figure 11: Comparison of tuned finite element and ROM frequency responses, for blade tip excitation with $C=4$.

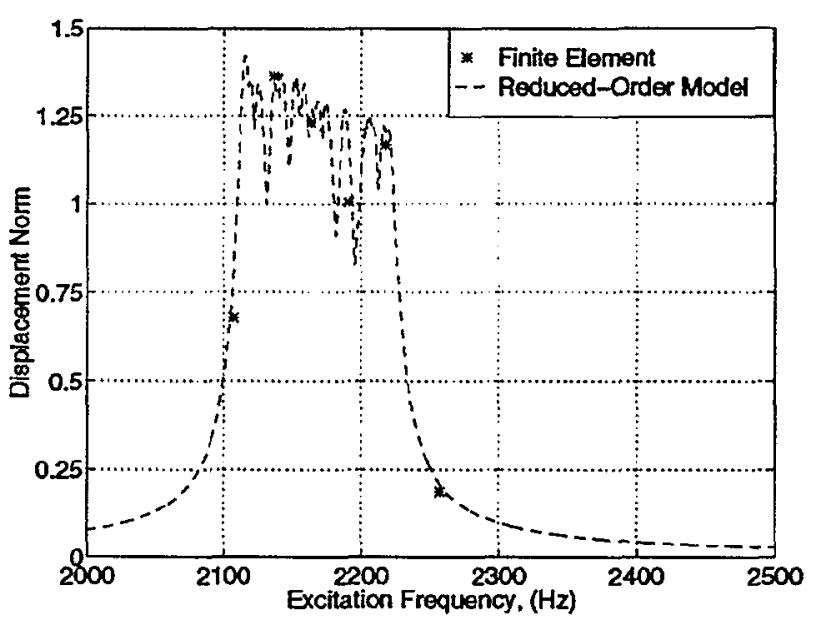

Figure 12: Comparison of mistuned finite element and ROM largest frequency responses, for blade tip excitation with $C=4$.
The matrix of aerodynamic coefficients used in Figure 13 may be obtained from any number of commercially available software packages. The codes typically use cyclic symmetry arguments to consider flow thru a cascade of cantilever blades. The coefficients obtained from the aeroelastic analysis are in the interblade phase angle coordinates and must be transformed to the modal coordinates of the cantilever blades for implementation into the ROM. The necessary transformation is given by:

$$
\mathbf{A}_{\mathrm{ROM}}=(\mathbf{E} \otimes \mathrm{I}) \mathbf{A}(\mathbf{E} \otimes \mathbf{I})^{T}
$$

where $\mathbf{A}$ is the matrix of aerodynamic coefficients in the interblade phase angle coordinates, $\mathbf{E}$ is the Fourier matrix, $I$ is the identity matrix, and $\otimes$ is the Kronecker product, defined in Appendix B. by:

The matrix of aerodynamic coefficients is given

$$
\mathbf{A}=\left[\begin{array}{ccccc}
\tilde{\mathbf{A}}_{\mathbf{0}} & \tilde{\mathbf{0}} & \tilde{\mathbf{0}} & \ldots & \tilde{\mathbf{0}} \\
\tilde{\mathbf{0}} & \tilde{\mathbf{A}}_{\mathbf{1}}^{\cos } & \tilde{\mathbf{0}} & \ldots & \tilde{\mathbf{0}} \\
\tilde{\mathbf{0}} & \tilde{\mathbf{0}} & \ddots & & \vdots \\
\vdots & \vdots & & \tilde{\mathbf{A}}_{\mathbf{2}}^{\text {sin }} & \tilde{\mathbf{0}} \\
\tilde{\tilde{0}} & \tilde{\mathbf{0}} & \ldots & \tilde{\mathbf{0}} & \tilde{\mathbf{A}}_{1}^{\text {sin }}
\end{array}\right]
$$

where $\overline{\mathbf{A}}_{\mathbf{n}}^{\text {cos }}$ and $\tilde{\mathbf{A}}_{\mathbf{n}}^{\text {sin }}$ are the matrices of aerodynamic coefficients corresponding to the $n$th harmonic, $n=0, \ldots, 14$. Note that $\mathbf{A}$ is block diagonal and that the size of each block is $M \times M$, where $M$ is the number of cantilever blade modes used in the aerodynamic analysis. The analysis presented in Figure 13 only includes aerodynamic terms for the first cantilevered blade mode shape coordinates.

Aerodynamic coefficients are in general complex, with the real part of the coefficients corresponding to added stiffness, and the imaginary part of the coefficients corresponding to (possibly negative) viscous damping. The results of an aeroelastic analysis for the case study rotor were not available. Coefficients, which are described below, were estimated based on prior experience with other rotors. The imaginary parts of the modal coefficients used to create Figure 13 assume that damping is uniform for each harmonic, and correspond to the addition of viscous damping of the same order of magnitude as the structural damping. The real parts of the modal coefficients are assumed to be zero, except for the coefficients correspanding to the engine order excitation. Since the rotor is subjected to engine order four excitation, weak, moderate, and strong aerodynamic couplings are defined by real, fourth harmonic coefficients that are $1 / 180,1 / 60$, and $1 / 36$ times smaller than the first cantilevered blade eigenvalue. 


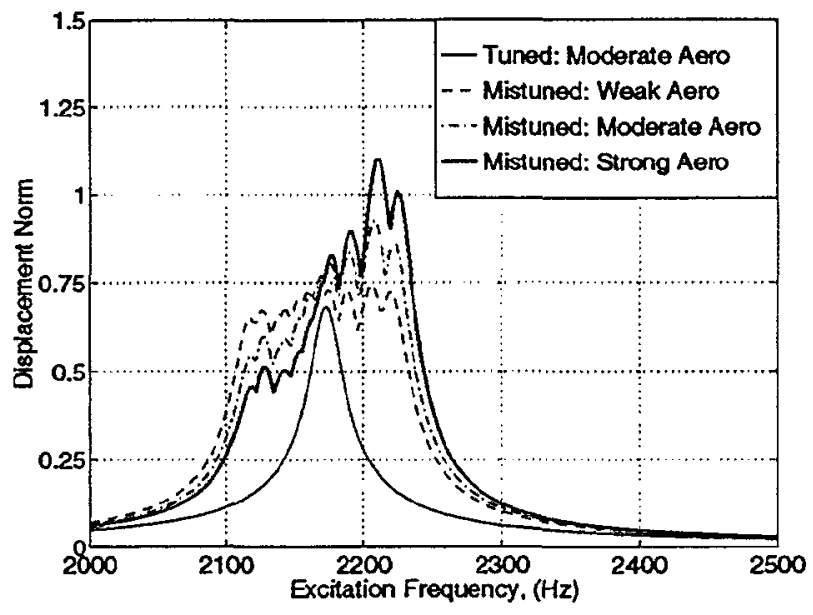

Figure 13: Comparison of tuned and mistuned largest frequency responses obtained with the ROM formulation for various aerodynamic coupling strengths, for blade tip excitation with $C=4$.

\begin{tabular}{|c|c|c|c|}
\hline & $\begin{array}{c}\text { ROM: } \\
\text { Weak } \\
\text { Aero- } \\
\text { dynamic } \\
\text { Coupling }\end{array}$ & $\begin{array}{c}\text { ROM: } \\
\text { Moderate } \\
\text { Aero- } \\
\text { dynamic } \\
\text { Coupling }\end{array}$ & $\begin{array}{c}\text { ROM: } \\
\text { Strong } \\
\text { Aero- } \\
\text { dynamic } \\
\text { Coupling }\end{array}$ \\
\hline Tuned & $\begin{array}{c}129.6 \\
10.7 \\
-10.6\end{array}$ & $\begin{array}{c}129.6 \\
10.7 \\
-10.6\end{array}$ & $\begin{array}{c}129.6 \\
10.7 \\
-10.6\end{array}$ \\
\hline Mistuned & $\begin{array}{c}144.8 \\
11.6 \\
-12.4\end{array}$ & $\begin{array}{c}184.7 \\
14.9 \\
-15.7\end{array}$ & $\begin{array}{c}219.9 \\
17.7 \\
-18.7\end{array}$ \\
\hline
\end{tabular}

Table 3: Comparison of tuned and mistuned ROM principal stresses (kpsi) at the largest resonant peak, for blade tip excitation with $C=4$. Principal stresses for the mistuned system can be as much as $70 \%$ higher than tuned system stresses.
Once the displacement field has been solved with the efficient ROM, displacements can be imported back into the finite element model for post processing of stress contours. Since all nodal displacements are prescribed, the finite element software merely performs the forward matrix calculations to obtain the stress field. Table 3 compares the resonant principal stresses obtained by tuned and mistuned reducedorder models with weak, moderate, and strong aerodynamic coupling. Principal stress values are listed for a node near the root of the blade and at the leading edge of the blade. Note that the principal stresses from the mistuned ROM are as much as $70 \%$ higher than the principal stresses from the tuned ROM. This $70 \%$ increase in principal stress corresponds to the $61 \%$ increase in resonant response amplitude observed in Fig. 13.

\subsection{Disk Structural Coupling}

The second form of interblade coupling represents the transmission of energy between blades through the disk. Figure 14 illustrates the tuned finite element and ROM frequency responses to an engine order one excitation. The resonance in Figure 14 corresponds to the second bending mode of a cantilever blade. It is located in the heart of an eigenvalue veering, between the second and third families of blade-dominated modes and the first family of disk-dominated modes, as can be seen in Fig. 4. As with the first bending mode, the correlation between tuned finite element and ROM responses in Fig. 14 is excellent.

Figure 15 depicts a comparison of mistuned finite element and ROM largest frequency responses for engine order one excitation. Mistuned finite element solutions are available at six selected frequencies as a spot check of the ROM response. Note that the mistuned response exceeds the tuned response by $44 \%$. Unlike the previous example, aerodynamic coupling is not included in the model, and disk structural coupling is the only form of interblade coupling included in Figs. 14 and 15. Figure 9 illustrates the mode shape corresponding to the resonant peak that yields a $44 \%$ increase in the forced response. Both finite ellement and ROM mode shapes correlate well in this frequency region.

Table 4 compares the resonant principal stresses obtained by finite element and reduced-order modeling. In general, principal stresses-obtained from the ROM are within $10 \%$ of the principal stresses determined with the finite element model. Note that the principal stresses for the mistuned finite element model are as much $53 \%$ higher than those for the tuned model. This $53 \%$ increase in principal stress 


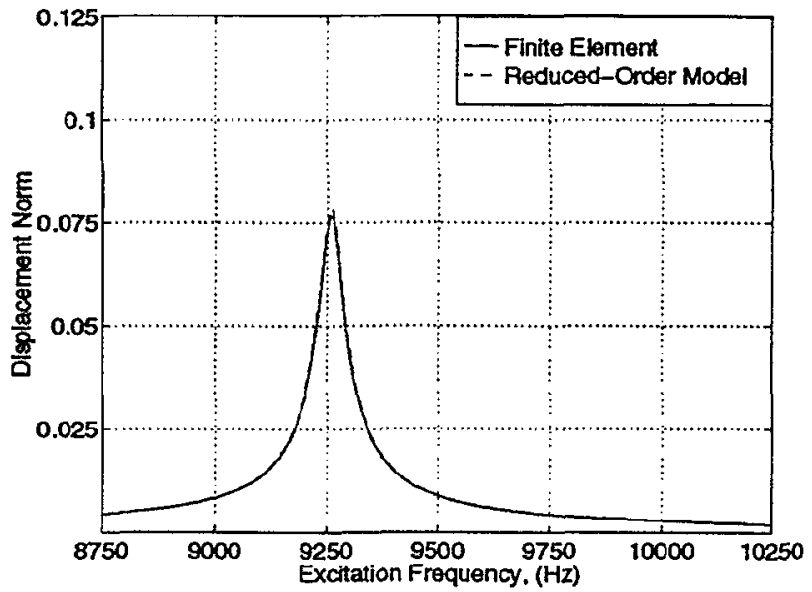

Figure 14: Comparison of tuned finite element and ROM frequency responses, for blade tip excitation with $C=1$.

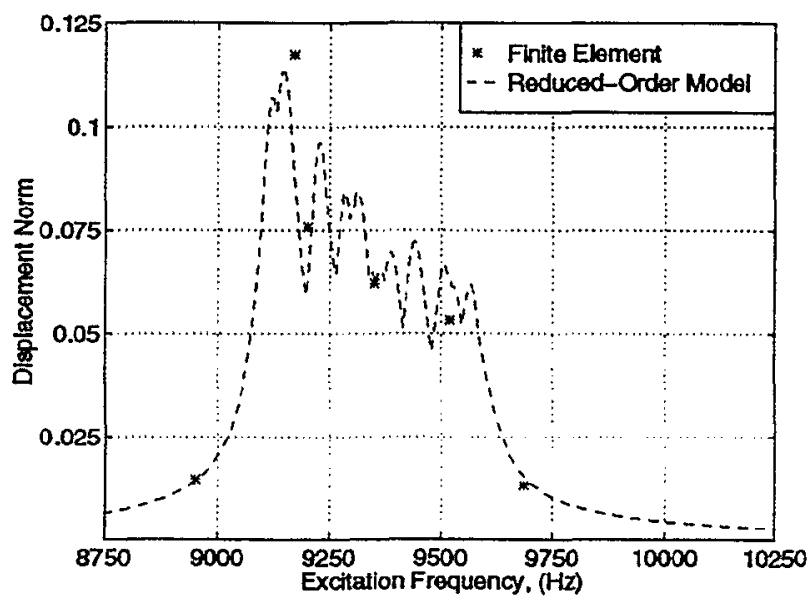

Figure 15: Comparison of mistuned finite element and $\mathrm{ROM}$ largest frequency responses, for blade tip excitation with $C=1$.

\begin{tabular}{|c|c|c||}
\hline & $\begin{array}{c}\text { Finite } \\
\text { Element }\end{array}$ & $\begin{array}{c}\text { Reduced-Order } \\
\text { Model }\end{array}$ \\
\hline \multirow{3}{*}{ Tuned } & 73.5 & $76.4(+4 \%)$ \\
& 2.6 & $4.5(+73 \%)$ \\
& -9.1 & $-8.5(+7 \%)$ \\
\hline \multirow{3}{*}{ Mistuned } & 112.7 & $108.9(-3 \%)$ \\
& 6.8 & $6.2(-9 \%)$ \\
& -14.2 & $-11.9(+17 \%)$ \\
\hline
\end{tabular}

Table 4: Comparison of finite element and ROM principal stresses (kpsi) at the largest resonant peak, for blade tip excitation with $C=1$. Percentage differences between the ROM and finite element results are given. Principal stresses for the mistuned system can be as much as $53 \%$ higher than stresses for the tuned system. Note that only structural coupling through the disk is considered, and that aerodynamic coupling is not included.

corresponds to the $44 \%$ increase in resonant response amplitude observed in Figs. 14 and 15.

\section{Numerical Simulations of Forced Response Statistics Using the ROM}

As the above free and forced response results illustrate, reduced-order models of bladed disks correlate well with much larger finite element models. The technique successfully captures and predicts mistuning effects on response amplitudes-something virtually impossible to achieve for most finite element models of industrial motors. More importantly, however, reduced-order modeling enables engineers to determine the statistical characteristics of blade forced response amplitudes for randomly mistuned rotors.

Figure 16 illustrates a Monte Carlo simulation of the statistics of the largest blade response amplitude, at any frequency in the range of 8,750 to 10,750 $\mathrm{Hz}$ (corresponding to the family of blade modes characterized by the second bending mode of a cantilevered blade), and for engine order one excitation. The simulation consists of frequency sweeps for 400 different mistuning patterns, obtained from a uniform distribution with $3 \%$ standard deviation. The tuned response, which is indicated in Figure 16, represents the optimal distribution of vibratory energy and clearly corresponds to the smallest response amplitude and the minimum stress state. Unfortunately, perfectly tuned turbomachinery rotors are not realizable, and the increase in blade response amplitude due to mistuning must be accounted for. Random mistuning must be compensated for by increasing the overall fatigue strength of the blades in order 
to meet some statistically determined stress level; Alternatively, designers could choose a deliberate mistuning pattern in an attempt to minimize the increase in mistuned vibratory stresses. In both instances, reduced-order modeling can aid the designer in capturing mistuning effects.

In Fig. 16, the 95th percentile of the largest blade response amplitude (the amplitude such that $95 \%$ of all mistuned rotors feature a smaller largest amplitude) is seen to correspond to a $53 \%$ increase over the tuned system's resonant response amplitude. The corresponding principle stresses, for one 95th percentile mistuning pattern, are: $\sigma_{1}=118.8$, $\sigma_{2}=6.8$, and $\sigma_{3}=-13.0 \mathrm{kpsi}$. Recall that the tuned principal stresses are: $\sigma_{1}=76.4, \sigma_{2}=4.5$, and $\sigma_{3}=-8.5 \mathrm{kpsi}$. Stresses for the $95 \mathrm{th}$ percentile of the largest response amplitude are thus as much as $56 \%$ higher than the tuned stresses. If the blisk design is based on tuned stress magnitudes, fatigue properties of the blades should be increased to compensate for the effect of random mistuning.

If compliance of $95 \%$ of the rotors is not acceptable, and 99th percentile, or even 99.9th percentile compliance is required, the computational cost of determining the statistics of forced response amplitudes with Monte Carlo simulations becomes a formidable task, even with the ROM. Analyses of an estimated 50,000 mistuned rotors would be required for the determination of 99.9 percentile amplitudes. Since the above 400 pattern simulation required two weeks of computational time on a 64 Mhz UNIX workstation, a new statistical analysis method is proposed, whereby the probability distributions of forced response amplitudes are approximated with statistical models.

Weibull distributions are frequently used to describe probabilistic engineering observations due to the versatility of the shape of the probability density function. The probability density function of a three-parameter Weibull distribution is given by: ${ }^{12,13}$

$$
f(x)=\frac{\beta}{\theta-\alpha}\left(\frac{x-\alpha}{\theta-\alpha}\right)^{\beta-1} e^{\left[-\left(\frac{x-\alpha}{\gamma-\alpha}\right)^{\alpha}\right]}
$$

where $\theta$ is the characteristic observation, $\beta$ is the slope, $\alpha$ is a threshold or location parameter, $x$ is the random variable under investigation, and $f(x)$ is its probability density function. To determine the correlation between Weibull estimates of response amplitudes and "true" response amplitudes, the largest blade response statistics from the Monte Carlo simulation were compared to Weibull approximations of the response amplitudes that utilize only $30 \mathrm{mis}-$ tuned rotors. This comparison is presented in Table 5 for the 5 th, 50 th, and 95 th percentiles of the largest response amplitude. Note that the threeparameter Weibull distribution closely approximate the "true" distribution.

Using Weibull distributions to calculate approximate largest forced response statistics, we investigated the effect of mistuning strength on the 5th, 50 th, and 95 th percentile responses. As shown in Figure 17, there is a maximum in the largest response amplitude for mistuning distributions with approximately $1 \%$ standard deviation. Principal stresses for one mistuning pattern corresponding to the 95th percentile response amplitude at $1 \%$ mistuning standard deviation are: $\sigma_{1}=141.8, \sigma_{2}=8.2$, and $\sigma_{3}=-15.4 \mathrm{kpsi}$. These principal stresses represent a $86 \%$ increase over the tuned principal stresses.

As mentioned earlier, deliberately mistuning the rotor is an alternative design strategy to statistically determining largest response amplitudes. When selecting a deliberate mistuning pattern, the mistuning strength should be significantly larger (say, by an order of magnitude) than the random mistuning associated with manufacturing and material tolerances, so as to provide a robust reduction of the amplitude increase due to mistuning. Table 6 lists a mistuning pattern with $10 \%$ standard deviation, for which the largest mistuned vibratory stresses are: $\sigma_{1}=85.7, \sigma_{2}=5.0$, and $\sigma_{3}=-9.2 \mathrm{kpsi}$. This deliberate mistuning pattern, which is indicated in Fig. 17, limits the principal stress increase to $12 \%$ over tuned levels. Thus, it is seen that deliberate mistuning can be effective in reducing forced response amplitude increases.

The choice of increasing the fatigue strength to some statistically determined amplitude, or deliberately mistuning the system, is up to the designer. But, it should be pointed out that a specific mistuning pattern may not reduce the vibratory stresses at other frequency regions of operation or in other modes of vibration. In fact, a specific mistuning pattern may result in even larger increases in vibratory stresses in other frequency regions. Statistically determining the maximum vibratory stress levels and increasing the fatigue strength of the individual blades is a more robust design strategy if the operational range of the rotor encompasses several modes of vibration.

\section{Conclusions}

Excessive vibratory-stress-levels is an industrywide problem faced by all turbomachinery manufactures. Small and unavoidable irregularities in the structural properties of individual blades may lead to qualitatively different dynamic behavior than that experienced by a perfectly tuned rotor. This mistun- 


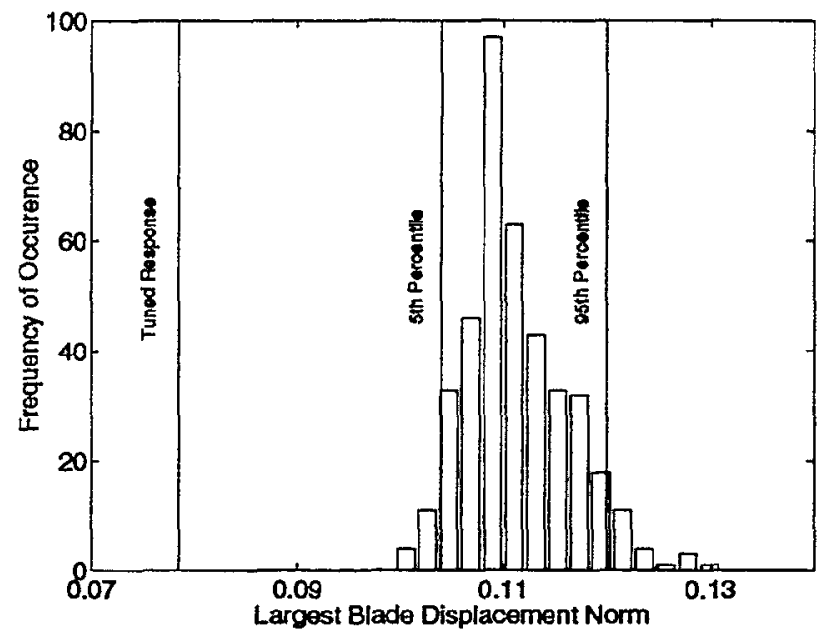

Figure 16: Histogram of the largest blade response amplitude for engine order one excitation. Obtained by Monte Carlo simulation of 400 different mistuned systems with uniform distributions of $3 \%$ standard deviation.

\begin{tabular}{||c|c|c|c||}
\hline \hline $\begin{array}{c}\text { Mistuning } \\
\text { Patterns }\end{array}$ & $\begin{array}{c}\text { 5th } \\
\text { Percentile }\end{array}$ & $\begin{array}{c}50 \text { th } \\
\text { Percentile }\end{array}$ & $\begin{array}{c}95 \text { th } \\
\text { Percentile }\end{array}$ \\
\hline $1-30$ & 0.1027 & 0.1116 & 0.1215 \\
& $(-1.2 \%)$ & $(+0.5 \%)$ & $(+1.2 \%)$ \\
\hline $31-60$ & 0.1018 & 0.1091 & 0.1221 \\
& $(-2.1 \%)$ & $(-1.7 \%)$ & $(+1.7 \%)$ \\
\hline $61-90$ & 0.1023 & 0.1102 & 0.1160 \\
& $(-1.7 \%)$ & $(-0.7 \%)$ & $(-3.4 \%)$ \\
\hline $91-120$ & 0.1039 & 0.1120 & 0.1195 \\
& $(-0.1 \%)$ & $(+0.9 \%)$ & $(-0.4 \%)$ \\
\hline $121-150$ & 0.1037 & 0.1107 & 0.1234 \\
& $(-0.3 \%)$ & $(-0.3 \%)$ & $(+2.8 \%)$ \\
\hline Monte Carlo & 0.1040 & 0.1110 & 0.1200 \\
\hline \hline
\end{tabular}

Table 5: Comparison of Weibull to Monte Carlo determined responses for 5 th, $50 \mathrm{th}$, and 95 th percentile largest blade response amplitudes. Percent discrepancies are indicated in parenthesis. The approximate response amplitudes from the Weibull distributions closely approximate the Monte Carlo determined responses.

\begin{tabular}{||c|c||}
\hline \hline Blade Number & Mistuning $\delta_{i}$ \\
\hline 1 & -0.118066 \\
2 & 0.172490 \\
3 & -0.015823 \\
4 & 0.094008 \\
5 & -0.031693 \\
6 & -0.026881 \\
7 & 0.055922 \\
8 & -0.103471 \\
9 & 0.154616 \\
10 & -0.098727 \\
11 & 0.040488 \\
12 & 0.022711 \\
13 & -0.044520 \\
14 & -0.102324 \\
15 & 0.009117 \\
16 & 0.145402 \\
17 & -0.095270 \\
18 & -0.172001 \\
19 & 0.114509 \\
20 & -0.153146 \\
21 & 0.049593 \\
22 & -0.080806 \\
23 & -0.107639 \\
24 & -0.111271 \\
25 & -0.120292 \\
26 & -0.054302 \\
27 & -0.029619 \\
28 & 0.144849 \\
29 & -0.153813 \\
\hline \hline
\end{tabular}

Table 6: Deliberate mistuning pattern with $10 \%$ standard deviation, which was found to limit the largest amplitude response to $10 \%$ over the tuned value. The corresponding principal stress increase is only $12 \%$ higher than the tuned principal stress. 


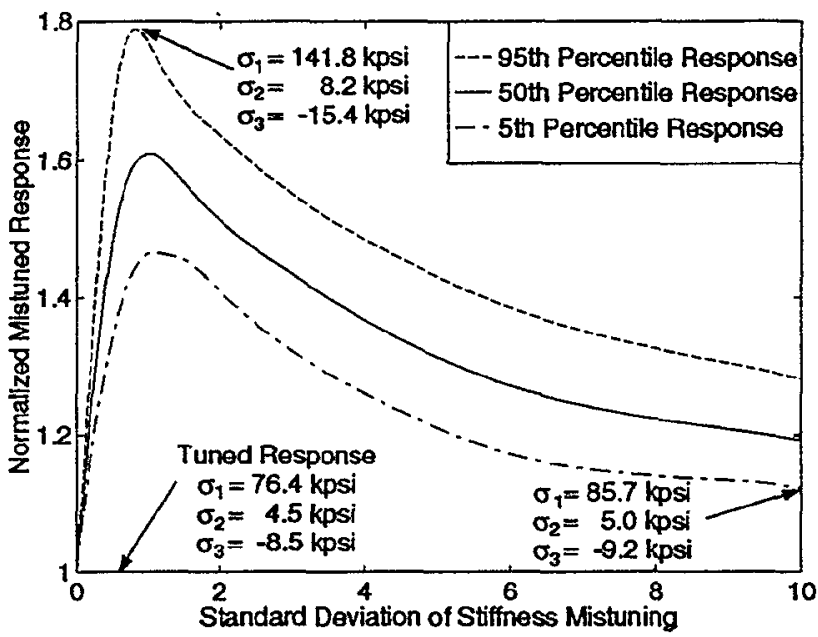

Figure 17: Variation in largest blade response amplitude with standard deviation of mistuning. Note that a maximum occurs for mistuning distributions with $1 \%$ standard deviation. Mistuned principal stresses are as much as $86 \%$ higher than the tuned principal stresses.

ing may confine vibrational energy to a single blade in the rotor, causing modes shapes to become spatially localized. This paper verifies the existence of localized modes in industrial turbomachinery rotors. Localized modes were found to exist in areas of high modal density, such as blade-dominated modes.

The existence of localized modes raises the possibility of significant amplitude increases in the forced response of the rotor. In particular, mistuned responses can greatly exceed tuned response levels if appropriate levels of interblade coupling are present. Two sources of interblade coupling, aerodynamic and disk structural coupling, were identified and examined for a case study rotor. Aerodynamic coupling was found to increase displacement and vibratory stress levels by $61 \%$ and $70 \%$, respectively, over tuned response levels. Disk structural coupling was found to increase displacement and vibratory stress levels by $44 \%$ and $53 \%$, respectively, over tuned response levels.

In all investigations, excellent correlation was observed between finite element and ROM responses. The computationally inexpensive ROM was used to determine statistically the variation in response amplitudes due to mistuning. For engine order one excitation, the 95th percentile principal stresses for a mistuned rotor can be as much as $86 \%$ higher than those for the tuned rotor. The design capabilities of the ROM were further highlighted by the determination of a deliberate mistuning pattern that limits the principal stress increase over tuned levels to only $12 \%$.

\section{Acknowledgements}

This work was sponsored by the GUIde Forced Response Consortium.

\section{References}

1 D. J. Ewins. "The Effects of Detuning Upon the Forced Vibrations of Bladed Disks". Journal of Sound and Vibration, 9(1):65-79, 1969.

2 D. J. Ewins. "Vibration Characteristics of Bladed Disk Assemblies". Journal Mechanical Engineering Science, 15(3):165-186, 1973.

3 H. Irretier. "Spectral Analysis of Mistuned Bladed Disk Assemblies by Component Mode Synthesis". In Proceedings of the 9th Biennial Conference on Mechanical Vibration and Noise of the Design and Production Engineering Technical Conferences, 1983.

${ }^{4}$ S. T. Wei and C. Pierre. "Localization Phenomena in Mistuned Assemblies with Cyclic Symmetry, Part I: Free Vibrations". ASME Journal of Vibration, Acoustics, Stress, and Reliability in Design, 110(4):429-438, 1988.

${ }^{5}$ S. T. Wei and C. Pierre. "Localization Phenomena in Mistuned Assemblies with Cyclic Symmetry, Part II: Forced Vibrations". ASME Journal of Vibration, Acoustics, Stress, and Reliability in Design, 110(4):439-449, 1988.

${ }^{6}$ G. S. Ottarsson, M. P. Castanier, and C. Pierre. "A Reduced-Order Modeling Technique for Mistuned Bladed Disks". In Proceedings of the 35th AIAA/ASME/ASCE/AHS/ASC Strutures, Structural Dynamics, and Materials Conference, 1994. Also, ASME Journal of Vibration and Acoustics, in print.

7 M. J. Kruse and C. Pierre. "Forced Response of Mistuned Bladed Disks Using Reduced-Order Modeling". In Proceedings of the 37th AIAA/ASME/ASCE/AHS/ASC Structures, Structural Dynamics, and Materials Conference, Salt Lake City, Utah, April 1996.

8 G. S. Ottarsson and C. Pierre. "On the Effects of Interblade Coupling on the Statistics of 
Maximum Forced Response Amplitudes in Mistuned Bladed Disks". In Proceedings of the 36th AIAA/ASME Strutures, Structural Dynamics, and Materials Conference, volume 95, page 1494, New Orleans, Louisiana, 1995.

9 Pierre C. "Mode Localization and Eigenvalue Loci Veering Phenomena in Disordered Structures". Journal of Sound and Vibration, 126(3):485-502, 1988.

10 J.A. Fabunmi. "Forced Vibration of a Single Stage Axial Compressor Rotor". ASME Journal of Engineering for Power, 102(2):322-329, 1980.

11 J. H. Griffin and T. M. Hoosac. "Model Development and Statistical Investigation of Turbine Blade Mistuning". ASME Journal of Vibration, Acoustics, Stress, and Reliability in Design, 106:204-210, 1984.

12 W. Nelson. Applied Life Data Analysis. Wiley, 1982.

13 J. F. Lawless. Statistical Models and Methods for Lifetime Data. Wiley, 1982.

\section{Appendix A: Nomenclature}

(Matrix dimensions in parenthesis)

$C \quad$ Engine order excitation

$E_{i} \quad$ Young's modulus of blade $i$

$E_{0} \quad$ Young's modulus for a tuned blade

$G \quad$ Modulus of rigidity

$G_{\text {Struc }}$ Structural damping coefficient

$j \quad$ Imaginary number $\equiv \sqrt{-1}$

$m_{b} \quad$ Number of cantilever blade modes

$m_{d} \quad$ Number of disk-induced static modes

$n \quad$ Harmonic number

$n_{F E} \quad$ Number of blade finite element degrees of freedom per sector

$N \quad$ Number of blades $(N=29)$

$M \quad$ Number of cantilever blade modes used in the aerodynamic analysis

$\delta_{i} \quad i$ th blade stiffness mistuning

$\rho \quad$ Mass density

$\nu \quad$ Poisson's ratio

$\zeta \quad$ Viscous damping ratio

$\phi_{i-} \quad$ Interblade phase angle, $i=1, \ldots, P$

$\omega \quad$ Excitation frequency

$\otimes \quad$ Kronecker product (See Appendix A) $a_{n} \quad$ Vector of generalized coordinates corresponding to the $n$ nodal diameter disk modes $\left(m_{d}, 1\right)$

a Vector of generalized coordinates for all disk modes, $\mathbf{a}=\left[\mathbf{a}_{\mathbf{0}}^{\mathbf{T}}, \mathbf{a}_{1}^{\mathbf{T}}, \ldots, \mathbf{a}_{\mathbf{p}}^{\mathbf{T}}\right]^{\mathbf{T}}$

$\mathbf{b}_{\mathbf{i}} \quad$ Vector of generalized coordinates corresponding to the ith blade's cantilevered modes $\left(m_{b}, 1\right)$

b Vector of generalized coordinates for all $N$ blades, $\mathbf{b}=\left[\mathbf{b}_{1}^{\mathbf{T}}, \mathbf{b}_{2}^{\mathbf{T}}, \ldots, \mathbf{b}_{\mathbf{N}}^{\mathbf{T}}\right]^{\mathbf{T}}\left(m_{b} N, 1\right)$

A Matrix of aerodynamic coefficients in the interblade phase angle coordinates $(M N, M N)$

$\tilde{\mathbf{A}}_{\mathbf{n}}^{\text {cos }}$ Cosinus portion of the $n$th harmonic matrix of aerodynamic coefficients in the interblade phase angle coordinates $(M, M)$

$\tilde{\mathbf{A}}_{\mathbf{n}}^{\text {sin }}$ Sinus portion of the $n$th harmonic man trix of aerodynamic coefficients in the interblade phase angle coordinates $(M, M)$

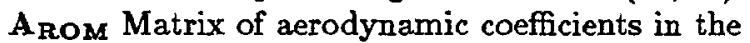
cantilevered blade modal coordinates

E Fourier matrix

$\tilde{\mathbf{f}}$ Force vector on a single blade in the finite element coordinates

F Forcing vector on the entire assembly of blades in the finite element coordinates

I Identity matrix

$\tilde{\mathbf{K}}_{\mathbf{b}} \quad$ Finite element stiffness matrix of a free blade $\left(n_{F E}, n_{F E}\right)$

$K_{b} \quad$ Blade portion of the stiffness matrix of the entire assembly $\left(n_{F E} N, n_{F E} N\right)$

$\hat{\mathbf{K}}_{\mathrm{b}} \quad$ Modal stiffness matrix of the entire blade assembly $\left(m_{b} N, m_{b} N\right)$

$\hat{\mathrm{K}}_{\mathbf{d}} \quad$ Modal stiffness matrix of the entire disk $\left(m_{d} N, m_{d} N\right)$

$\tilde{\mathbf{M}}_{\mathrm{b}} \quad$ Finite element mass matrix of a single blade $\left(n_{F E}, n_{F E}\right)$

$\mathbf{M}_{\mathrm{b}} \quad$ Blade portion of the mass matrix of the entire assembly $\left(n_{F E} N, n_{F E} N\right)$

$\tilde{\mathbf{u}}_{\mathbf{i}}^{\mathbf{b}} \quad$ ith cantilever blade mode for one blade $\left(n_{F E}, 1\right)$

$U^{\text {b }} \quad$ Cantilever blade modes for the entire assembly $\left(n_{F E} N, m_{b} N\right)$

$\tilde{\mathbf{u}}_{\mathbf{n}, \mathbf{m}}^{\mathbf{d}}, \tilde{\mathbf{u}}_{\mathbf{n}, \mathbf{m}}^{\mathbf{d}^{*}}$ Complex conjugate pair of diskinduced modes, $n$th harmonic, $m$ th mode $\left(n_{F E}, 1\right)$

$\mathbf{U}_{\mathbf{n}}^{\mathbf{d}} \quad$ Matrix of $n$th harmonic disk-induced modes for the entire assembly $\left(n_{F E} N, m_{d}\right)$

$U^{d} \quad$ Matrix of disk-induced modes for the entire assembly $\left(n_{F E} N, m_{d} N\right)$ 


\section{Appendix B}

The aerodynamic matrix can be transformed from the interblade phase angle coordinates to the modal coordinates of the cantilever blades by the Fourier matrix. The complex form of the Fourier matrix is:

$$
\begin{gathered}
\mathrm{E}=\left(e_{k i}\right) \quad k, i=1, \ldots, N \\
e_{k i}=\frac{1}{\sqrt{N}} e^{\frac{j 2 \pi(i-1)(k-2)}{N}} \quad k, i=1, \ldots, N
\end{gathered}
$$

The real-valued form of Eq. (B.1) is:

$$
\underline{e}_{k i}=\left\{\begin{array}{cll}
\frac{1}{\sqrt{N}} & \text { if } & i=1 \\
\sqrt{\frac{2}{N}} \cos \left(\frac{2 \pi(i-1)(k-1)}{N}\right) & \text { if } & 1<i<P \\
\frac{-1^{k-1}}{\sqrt{N}} & \text { if } & i=P, \\
\sqrt{\frac{2}{N}} \sin \left(\frac{2 \pi(i-1)(k-1)}{N}\right) & \text { if } & P<i \leq N \\
k=1, \ldots, N &
\end{array}\right.
$$

The Kronecker product of two matrices is

$$
\mathbf{C}=\mathbf{A} \otimes \mathbf{B}=\left[\begin{array}{cccc}
a_{11} \mathbf{B} & a_{12} \mathrm{~B} & \ldots & a_{1 N} \mathbf{B} \\
a_{21} \mathrm{~B} & a_{22} \mathrm{~B} & \ldots & a_{2 N} \mathrm{~B} \\
\vdots & \vdots & \ddots & \vdots \\
a_{N 1} \mathrm{~B} & a_{N 2} \mathrm{~B} & \ldots & a_{N N} \mathrm{~B}
\end{array}\right]
$$

